EDITOR-IN-CHIEF

Annalisa Bossi

Università Ca’ Foscari di Venezia

Dipartimento di Informatica

Via Torino 155

I-30172 Mestre-Venezia, ITALY

EMAIL bossi@dsi.unive.it
FOUNDING EDITOR-IN-CHIEF

Jack Minker

Department of Computer Science

and Institute for Advanced Computer Studies,

University of Maryland,

College Park, Maryland 20742, USA,

EMAIL minker@cs.umd.edu

\author{
AREA EDITORS \\ Constraints \\ Peter J. Stuckey \\ University of Melbourne \\ pjs@cs.mu.oz.au \\ Databases and Semantic Web Reasoning \\ Michael Kifer \\ Stony Brook University \\ kifer@cs.stonybrook.edu \\ Design, Analysis and Implementation of \\ Languages \\ David S. Warren \\ Stony Brook University \\ warren@cs.sunysb.edu \\ Inductive Logic Programming and Multi- \\ relational Data Mining \\ Luc De Raedt \\ Katholieke Universiteit Leuven \\ luc.deraedt@cs.kuleuven.be
}

Knowledge Representation and

Nonmonotonic Reasoning

Michael Gelfond

Texas Tech. University

mgelfond@cs.ttu.edu

Logic Programming Methodology \&

Applications

Gopal Gupta

The University of Texas at Dallas

gupta@utdallas.edu

Specification, Analysis and Verification of

Systems

Michael Leuschel

University of Düsseldorf

leuschel@cs.uni-dusseldorf.de
Theory

Giorgio Levi

Universitá di Pisa

levi@di.unipi.it

Technical Notes

Catuscia Palamidessi

INRIA Futurs and LIX

catuscia@lix.polytechnique.fr

Programming Pearls

Lee Naish

University of Melbourne

lee@cs.mu.oz.au

Bart Demoen

Katholieke Universiteit Leuven

Bart.Demoen@cs.kuleuven.be

Book Reviews

Krzysztof Apt

CWI, The Netherlands

apt@cwi.nl

\section{EDITORIAL ADVISORS}

C. Baral

Arizona State University

Maurice Bruynooghe

Katholieke Universiteit

Leuven

M. Carlsson

Swedish Institute of

Computer Science

A. Colmerauer

Université d'Aix Marseille II

V. Dahl

Simon Fraser University

D. De Schreye

Katholieke Universiteit

Leuven

J. Dix

Clausthal University of

Technology, Germany

P.M. Dung

Asian Institute of

Technology, Thailand

T. Eiter

Vienna University of

Technology
P. Flach

University of Bristol

M. Gabbrielli

University of Bologna

J. Gallagher

Roskilde University

Manuel Hermenegildo

Universidad Politecnica

de Madrid

K. Inoue

National Institute of

Informatics, Japan

Joxan Jaffar

National University of

Singapore

A. Kakas

University of Cyprus

A. King

University of Kent

R.A. Kowalski

Imperial College

L. Libkin

University of Toronto
V. Lifschitz

University of Texas at

Austin

M. Maher

National ICT

Australia

D. Miller

INRIA, École

Polytechnique

S. Muggleton

Imperial College

G. Nadathur

University of Minnesota

I. Niemela

Helsinki University of

Technology

R. O'Keefe

University of Otago, New

Zealand

L.M. Pereira

Universidade Nova de

Lisboa

J.A. Robinson

Highland Institute

\section{INTERNET ACCESS}

The journal is included in the Cambridge Journals Online service which can be found at journals.cambridge.org/.

Refer also for other information to the dedicated journal web site at http//: www.cs.kuleuven.be/ dtai/projects/ALP 